\title{
ESTUDO DA RELAÇÃO ENTRE O COMPRIMENTO DA BANDA ILIOTIBIAL E O DESALINHAMENTO PÉLVICO
}

\author{
FARIA CDCM, Lima FFP E TEIXEIRA-SALMELA LF \\ Departamento de Fisioterapia, Escola de Educação Física, Fisioterapia e Terapia Ocupacional, Universidade Federal de \\ Minas Gerais, Belo Horizonte, MG - Brasil \\ Correspondência para: Luci Fuscaldi Teixeira-Salmela, Ph.D., Departamento de Fisioterapia, Universidade Federal de \\ Minas Gerais, Av. Antônio Carlos, 6627, Campus Pampulha, CEP 31270-901, Belo Horizonte, MG - Brasil, \\ e-mail: lfts@ufmg.br
}

Recebido: 04/04/2005 - Revisado: 27/09/2005 - Aceito: 20/04/2006

\begin{abstract}
RESUMO
Contextualização: Desalinhamentos pélvicos, como a inclinação lateral, ocasionam diferentes disfunções e queixas de dor. Na literatura, são apontados diversos fatores associados à inclinação lateral da pelve, sendo um deles o comprimento da banda iliotibial. Objetivo: Verificar a existência de correlação entre o comprimento da banda iliotibial e o desalinhamento pélvico no plano frontal. Método: Trinta e dois indivíduos (22,5 \pm 2,6 anos) de ambos os sexos, assintomáticos, sem diferença no comprimento dos membros inferiores e sem torção pélvica, foram incluídos no estudo. Para a medida do comprimento da banda iliotibial, utilizou-se o teste de Ober modificado, um nível pélvico e um inclinômetro e, para determinar o desalinhamento pélvico no plano frontal, foram utilizadas medidas de estruturas pélvicas obtidas por um antropômetro com o indivíduo posicionado em um instrumento de suporte de padronização. O Coeficiente de correlação de Pearson foi utilizado para investigar a associação entre as variáveis analisadas: o desalinhamento pélvico no plano frontal, determinado pela diferença da altura da espinha ilíaca ântero-superior mais elevada menos a altura da mais baixa e o comprimento da banda iliotibial, determinado pela diferença entre o comprimento da banda iliotibial correspondente à espinha ilíaca ântero-superior mais elevada menos o comprimento da mesma estrutura correspondente a espinha mais baixa. Resultados: Não foi encontrada correlação estatisticamente significativa entre as variáveis analisadas $(r=0,12, p=0,53)$. Conclusão: Os achados do presente estudo indicam que o comprimento da banda iliotibial, isoladamente, parece não influenciar o alinhamento pélvico no plano frontal. Entretanto, outros fatores podem estar envolvidos e precisam ser identificados.
\end{abstract}

Palavras-chave: Banda iliotibial, avaliação, teste de Ober, desalinhamento pélvico.

\section{ABSTRACT \\ Study on the Relationship Between Iliotibial Band Length and Pelvic Misalignment}

Background: Pelvic misalignments such as lateral pelvic tilt may lead to dysfunctions and pain complaints. Studies in the literature have indicated several factors associated with lateral pelvic tilt, and one of these is the length of the iliotibial band. Objective: Investigate the presence of an association between iliotibial band length and frontal plane pelvic tilt. Method: Thirty-two asymptomatic individuals of both sexes (22.5 \pm 2.6 years), without leg-length differences or pelvic torsion, were included in the study. The modified Ober test, performed with a pelvic level and an inclinometer, was used to assess the iliotibial band length. To determine the pelvic misalignment in the frontal plane, the individual was positioned on standard support equipment and pelvic structure measurements were obtained using an anthropometer. Pearson's correlation coefficient was utilized to determine the degree of association between pelvic misalignment in the frontal plane (established as the difference in height between higher and lower anterosuperior iliac spines) and the length of the iliotibial band (established as the difference in iliotibial band lengths corresponding to the higher and lower anterosuperior iliac spines). Results: No statistically significant correlations were found between the variables analyzed $(r=0.12 ; p=0.53)$. Conclusion: The present findings indicate that iliotibial band length alone does not appear to influence frontal plane pelvic alignment. Other factors may be involved and these still need to be identified.

Key words: iliotibial band, assessment, Ober test, pelvic misalignment. 


\section{INTRODUÇÃO}

O posicionamento de todas as articulações e estruturas do corpo, em um dado momento, compõe a postura ${ }^{1}$, sendo a posição da pelve $\mathrm{e}^{1,2,3}$ e o equilíbrio entre a musculatura que cruza as articulações ${ }^{1}$ essenciais para um bom alinhamento postural. Em uma situação de cadeia cinemática fechada, como na postura em pé, o posicionamento de um segmento influenciará no posicionamento de segmentos adjacentes. Como a pelve é considerada uma estrutura chave no alinhamento do corpo ${ }^{1,2,3}$, qualquer alteração da sua posição neutra causará movimentos compensatórios em várias regiões, sendo a coluna lombar e o quadril as primeiras a serem afetadas $^{3}$, ocasionando síndromes de uso excessivo e sintomas de dor ${ }^{2}$, principalmente na coluna, quadril e joelho ${ }^{3}$.

Diversos fatores, tanto estruturais quanto funcionais, podem estar associados à inclinação lateral da pelve ${ }^{4}$, como escoliose $^{5}$, diferença no comprimento dos membros inferiores $^{1,2}$, pé pronado ${ }^{6}$, desequilíbrios musculares ${ }^{1,2,7}$, encurtamento dos músculos que se originam ou se inserem nos ossos pélvicos ${ }^{1,2,3,6,7}$, dentre outros. A diferença no comprimento dos membros inferiores é o fator mais citado na literatura como associado à inclinação pélvica lateral ${ }^{8}$. Entretanto, na maior parte dos casos, essa diferença não é real, mas sim aparente, pois, na realidade, está associada a uma fraqueza ou a um encurtamento dos músculos abdutores do quadril ${ }^{7}$. Nessa situação, existe um fator funcional que leva à inclinação lateral da pelve ${ }^{4}$.

O encurtamento da banda iliotibial (BIT) é citado na literatura como um dos fatores funcionais associados à inclinação pélvica lateral ${ }^{1,2,7,8}$. A BIT é definida como um espessamento lateral da fáscia lata, que se estende da crista ilíaca à tuberosidade lateral da tíbia, cabeça da fíbula, côndilo lateral do fêmur e septo intermuscular entre os músculos isquiossurais ${ }^{9,10}$. As fibras da BIT apresentam conexões com diversas estruturas, como músculos glúteo máximo e tensor da fáscia lata ${ }^{10,11}$ e retináculo patelar lateral ${ }^{10,12}$, uma expansão aponeurótica do tendão do músculo vasto lateral ${ }^{10}$. Por se fixar em um osso pélvico, um encurtamento unilateral dessa estrutura apresenta potencial de produzir uma inclinação pélvica lateral para o mesmo lado ${ }^{1,2,7}$.

Apesar de alguns autores afirmarem a presença de uma associação entre o encurtamento da BIT com a inclinação pélvica lateral para o lado da estrutura encurtada, considerarem essa uma alteração comum nos indivíduos ${ }^{1,7}$ e apontarem diversas compensações ${ }^{3}$, disfunções ${ }^{7,13,14,15}$ e queixas de dor altamente prevalentes ${ }^{2,16,17}$ associadas à inclinação lateral da pelve, não foi encontrado nenhum estudo que investigasse essa relação. Assim, o objetivo deste estudo foi verificar a existência de correlação entre o comprimento da banda iliotibial (CBIT) e o desalinhamento pélvico (DP) no plano frontal.

\section{METODOLOGIA}

\section{Amostra}

Para a realização deste estudo foram recrutados, na comunidade, indivíduos de qualquer sexo, com idade variando entre 18 e 30 anos. Foram excluídos do estudo indivíduos que apresentaram qualquer um dos seguintes critérios: nível de compreensão insuficiente para realização do teste; obesidade; patologia ou limitação física que impedisse a execução dos movimentos necessários à avaliação; alterações congênitas no tronco e nos membros inferiores; história prévia de cirurgia na coluna e/ou membros inferiores; quadro álgico ou qualquer sinal inflamatório em estruturas dos membros inferiores no momento da avaliação; escoliose e/ou torção pélvica; assimetrias no contorno da superfície da coxa, joelho e perna; e diferença real na medida do comprimento dos membros inferiores. Todos assinaram um termo de consentimento livre e esclarecido aprovado pelo Comitê de Ética em Pesquisa da Universidade Federal de Minas Gerais, sob parecer de número ETIC 337/03.

\section{Procedimentos}

Inicialmente, foi realizada uma seleção dos voluntários por entrevista e avaliação clínica individual para determinar se o indivíduo preenchia os critérios de inclusão estabelecidos. Os dados foram coletados por dois pesquisadores devidamente treinados para realizar os procedimentos e, durante toda a coleta, um examinador ficou responsável pelo posicionamento do indivíduo, enquanto a medida era obtida pelo outro. Um estudo piloto foi realizado para determinar a confiabilidade teste-reteste das medidas, e altos índices de coeficiente de correlação intraclasse foram encontrados (CCI=0,99).

\section{Medida do comprimento real dos membros inferiores}

Para que o indivíduo fosse incluído no estudo, foi realizada uma medida do comprimento real dos seus membros inferiores, utilizando uma fita métrica de plástico padrão, graduada em milímetros, uma maca e uma caneta para demarcar as estruturas ósseas palpadas, conforme procedimentos válidos descritos por Beattie et al. ${ }^{18}$. Foram realizadas duas medidas em cada membro, e a subtração da média das medidas em um membro pela média do membro oposto correspondeu à diferença real no comprimento dos membros $^{5,18,17}$.

\section{Medida da torção e da inclinação pélvica}

Para avaliação da torção pélvica, foi utilizado um método não invasivo que, a partir de medidas das proeminências ósseas, fornece valores que permitem o cálculo trigonométrico para se obter o ângulo da inclinação de cada hemipelve no plano sagital $^{19}$, podendo, também, fornecer valores para se determinar a inclinação pélvica no plano frontal (figura 1). 


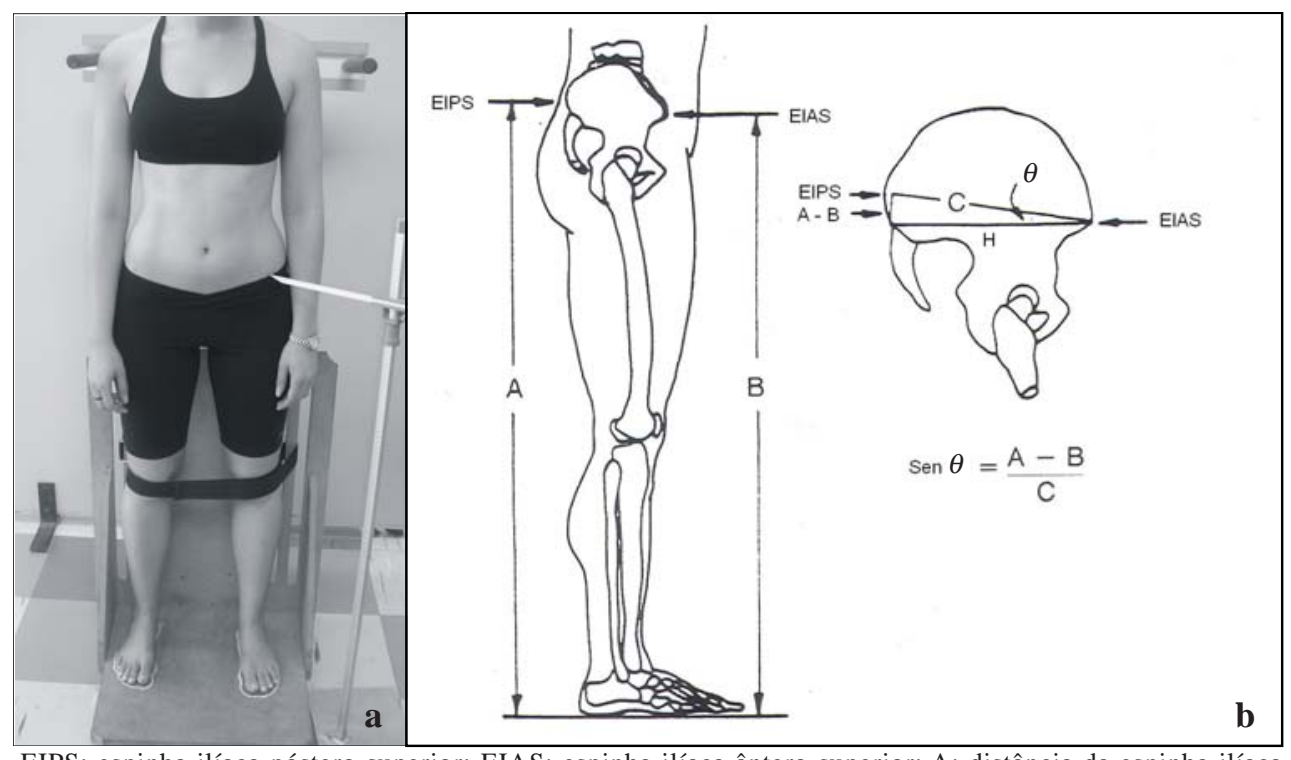

EIPS: espinha ilíaca póstero-superior; EIAS: espinha ilíaca ântero-superior; A: distância da espinha ilíaca póstero-superior ao solo; B: distância da espinha ilíaca ântero-superior ao solo; C: distância entre a espinha ilíaca póstero-superior e a espinha ilíaca ântero-superior; H: outro cateto do triângulo retângulo; $\theta$ : ângulo formado entre C e $\mathrm{H}$ - grau de inclinação da hemipelve no plano sagital.

Figura 1. a) Obtenção das medidas antropométricas da pelve; b) Diagrama do cálculo trigonométrico da inclinação pélvica no plano sagital (Adaptada de Sanders e Stavrakas, 1981, p. 49 ${ }^{19}$ ).

Esse método foi proposto em 1981 por Sanders e Stavrakras ${ }^{19}$ para medir o ângulo da inclinação pélvica na posição em pé e vem sendo utilizado em vários estudos, os quais obtiveram medidas confiáveis e representativas da inclinação pélvica ${ }^{20,21,22}$.

Como ilustrado na figura 1.b, considerando uma hemipelve, chamaremos de $\mathbf{C}$ a linha que representa a distância entre a sua espinha ilíaca ântero-superior (EIAS) e espinha ilíaca póstero-superior (EIPS), de A a linha que representa a distância da sua EIPS ao solo, de $\mathbf{B}$ a linha que representa a distância da sua EIAS ao solo e de $\mathbf{H}$ uma linha horizontal que passe pela sua espinha ilíaca mais inferior (geralmente a EIAS). Chamaremos de C', A', B' e $\mathbf{H}^{\prime}$ as linhas correspondentes às mesmas estruturas da hemipelve oposta. As linhas $\mathbf{C}, \mathbf{H}$ e aquela que corresponde à diferença entre A e B (A-B) ou as linhas C', H' e A'-B' formam um triângulo retângulo, sendo $\boldsymbol{\theta}$ ou $\boldsymbol{\theta}^{\prime}$ o ângulo formado entre $\mathbf{C}$ e $\mathbf{H}$ ou entre $\mathbf{C}^{\prime}$ e $\mathbf{H}^{\prime}$, respectivamente. Esse ângulo ( $\boldsymbol{\theta}$ ou $\left.\boldsymbol{\theta}^{\prime}\right)$ corresponde ao grau de inclinação da hemipelve no plano sagital. Portanto, se o ângulo obtido pelas medidas de uma hemipelve for o mesmo obtido pelas medidas da outra hemipelve (ou seja, $\boldsymbol{\theta}=\boldsymbol{\theta}^{\prime}$ ), pode-se concluir que não há torção pélvica. Isso irá ocorrer quando os valores de $\mathbf{C}$ forem iguais ao de $\mathbf{C}^{\prime}$ e os valores de $\mathbf{A}-\mathbf{B}$ forem iguais ao de $\mathbf{A}^{\prime}-\mathbf{B}^{\prime}$. Entretanto, os valores de $\mathbf{A}$ e $\mathbf{B}$ podem ser diferentes dos valores de A' e B', mas A-B pode ser igual a A'-B'. Nesse caso, teremos um DP e ausência de torção pélvica. $\mathrm{O}$ valor desse desalinhamento é dado pela subtração de $\mathbf{A}$ por $\mathbf{A}^{\prime}$ ou de $\mathbf{B}$ por $\mathbf{B}$.

Para coletar os valores de C, C', A, A', B e B', foi utilizado um antropômetro da marca GPS e um instrumento de suporte para padronização (ISP). O indivíduo foi instruído a retirar seus calçados e meias e se posicionar de forma ereta sobre o ISP. Os pés foram separados pela mesma distância presente entre as EIAS ${ }^{23}$, medida pela fita métrica. Em seguida, os pés foram colocados na posição neutra da articulação subtalar para que alterações dessa região não afetassem a altura das estruturas pélvicas. Para tal posicionamento, foi realizada a palpação da congruência talar de acordo com procedimentos descritos por Gould III $^{24}$. Uma fita de velcro foi colocada e ajustada em torno da tuberosidade da tíbia com a finalidade de se estabilizar a articulação do joelho. Para o controle da posição do tronco, foram colocadas duas referências horizontais $3 \mathrm{~cm}$ abaixo do acrômio. O antropômetro foi posicionado de um lado para se obter as distâncias entre a EIAS e a EIPS e entre as espinhas (EIAS e EIPS) e o solo e, em seguida, foi posicionado para se obter as mesmas medidas do outro lado (figura $1 \mathrm{a}$ ).

Para a medida da inclinação pélvica no plano frontal, foram utilizados os dados coletados na medida da inclinação pélvica no plano sagital. A diferença entre as medidas das alturas das EIAS foi considerada como valor da inclinação lateral da pelve. Um estudo utilizando o método trigonométrico de cálculo da inclinação pélvica com esses instrumentos obteve valores aceitáveis de confiabilidade das medidas obtidas ${ }^{22}$.

Considerando ser essa uma medida indireta de estruturas e, portanto, sujeita a uma determinada variabilidade, foi estipulada, como ausência de torção pélvica, uma diferença entre os valores de A - B e A' - B' menor ou igual a $0,5 \mathrm{~cm}$. Esse valor foi determinado durante o estudo piloto, uma vez que não foram encontradas, na literatura pesquisada, faixas 
de referência para se considerar ausência ou presença de torção pélvica. Assim, o indivíduo com ausência de torção pélvica, considerando os valores pré-estabelecidos e com características adequadas aos critérios de inclusão e exclusão citados anteriormente, participou da próxima etapa do estudo.

\section{Medida do comprimento da banda iliotibial}

Foram utilizados os procedimentos e instrumentos propostos por Melchione e Sullivan ${ }^{25}$, que encontraram valores de confiabilidade aceitáveis para as medidas intra-examinador $(\mathrm{CCI}=0,94)$ e interexaminador $(\mathrm{CCI}=0,73)$. Esses instrumentos foram: o teste de Ober modificado, um nível pélvico devidamente construído para a realização deste estudo segundo descrições de Melchione e Sullivan ${ }^{25}$, dois goniômetros universais e um inclinômetro da marca Starret Ind. \& Com. LTDA, São Paulo, graduado em um grau, devidamente calibrado pelo fabricante e com uma base constituída de imã, sob a qual foi acoplada uma régua de metal, o que permitiu melhor controle do seu posicionamento no segmento. É importante ressaltar que o teste de Ober modificado, assim como o teste de Ober original, são as ferramentas disponíveis, até o presente momento para se medir o comprimento da $\mathrm{BIT}^{13}$, sendo testes clássica e corriqueiramente utilizados, aceitos e recomendados para se obter o comprimento dessa estrutura $^{9,25,26,27}$.

O examinador 1 realizou a palpação e a demarcação das EIPS, alinhou o nível pélvico sobre os pontos marcados e o fixou com uma fita adesiva antialérgica. O indivíduo foi posicionado em decúbito lateral, com o membro infralateral em flexão de joelho, quadril e lombossacra até que a lordose lombar parecesse retificada. Esse mesmo examinador posicionou a pelve de forma a obter adequado alinhamento determinado pelo nível pélvico. Utilizando os dois goniômetros, o examinador 2 posicionou o quadril a $5^{\circ}$ de extensão e o joelho a $5^{\circ}$ de flexão, fixando-os com velcro para manutenção da posição. O indivíduo foi instruído a relaxar o membro inferior que estava sendo avaliado para que o examinador 2 o colocasse em completa abdução, deixando-o, em seguida, cair a favor da gravidade, até que o quadril não pudesse mais ser movimentado, tensionando a BIT. O mesmo examinador colocou a régua com o inclinômetro em contato com o eixo no goniômetro posicionado no joelho e, após estabilização do ponteiro do equipamento, realizou a leitura e o registro do grau de angulação entre o membro e a horizontal ${ }^{25}$. Durante todo esse procedimento, o examinador 1 assegurou a manutenção da pelve em neutro, segundo o nível pélvico, e a manutenção da articulação do quadril do membro avaliado em posição neutra de rotação, visualmente. Para cada membro foram realizadas duas medidas, e a média das medidas utilizada para análise.

\section{Análise estatística}

Estatísticas descritivas e testes de normalidade (ShapiroWilk) foram realizados para todas as variáveis, utilizando o pacote estatístico SPSS (versão 10.7 SPSS Inc.). Coeficientes de correlação intraclasse foram utilizados para determinar a confiabilidade teste-reteste das medidas. Para verificar a relação entre o CBIT e o DP, coeficientes de correlação de Pearson foram calculados entre as seguintes variáveis: diferença entre a altura da EIAS mais elevada e mais baixa (EIASe - EIASb) e diferença entre o CBIT do lado correspondente à EIAS mais elevada e do lado correspondente à mais baixa (CBITe - CBITb). O nível de significância estabelecido foi de $\boldsymbol{\alpha}<0,05$.

\section{RESULTADOS}

Foram recrutados 44 indivíduos, dos quais dois apresentavam diferença no comprimento dos membros inferiores e 10, torção pélvica, considerando a faixa estabelecida. Portanto, foram incluídos 32 indivíduos, com média de idade de 22,5 \pm 2,06 anos, 19 do sexo feminino e 13 do masculino, sendo 17 sedentários.

A tabela 1 apresenta as médias, os desvios-padrão e as faixas de variação de todas as medidas necessárias para análise da correlação entre as variáveis investigadas. Não foi detectada associação significativa entre a diferença da altura da EIAS mais elevada menos a altura da mais baixa com o CBIT do lado correspondente à EIAS mais elevada menos o CBIT correspondente à mais baixa $(r=0,12, p=0,531)$.

\section{DISCUSSÃO}

A premissa de que desordens biomecânicas na região pélvica podem causar assimetrias no posicionamento da pelve ainda não está completamente esclarecida. Uma explicação plausível relatada para a assimetria do posicionamento pélvico é a disfunção dos tecidos moles ${ }^{28}$, com destaque para a BIT. É reportada na literatura, de forma teórica, a presença de uma relação entre o CBIT e o $\mathrm{DP}^{1,2,7,8}$, e esse é um raciocínio comumente utilizado na prática clínica. Entretanto, não foi encontrado nenhum estudo científico que investigasse essa relação. Neste estudo, a existência de uma correlação entre o CBIT e o DP não foi confirmada.

A única forma descrita na literatura para se avaliar clinicamente o CBIT é por meio dos testes de Ober ou Ober modificado ${ }^{1}$. No presente estudo, utilizando os procedimentos descritos por Melchione e Sullivan ${ }^{25}$, foi obtido CCI intra-examinador de 0,99 , superior aos valores previamente reportados $^{10,12,25}$. Os testes de Ober e Ober modificado, desde que foram propostos, vêm sendo utilizados na clínica e em pesquisas como forma de se medir o $\mathrm{CBIT}^{1,10,12,25}$ e, com o controle de alguns fatores, esses são os melhores meios de se avaliar o $\mathrm{CBIT}^{12,25}$. Entretanto, não foi encontrado nenhum estudo sobre a validade concorrente desses testes, provavelmente pela dificuldade de se obter diretamente e de forma quantitativa o comprimento de estruturas musculotendíneas in vivo. 
Para se obter medidas de alinhamento pélvico não há método considerado padrão-ouro ${ }^{21}$. Técnicas objetivas, como a radiografia, a cintilografia e a tomografia computadorizada, poderiam ser utilizadas para fornecer imagens da pelve $e^{5,19,20}$. Entretanto, para se obter as medidas necessárias para este estudo (torção pélvica e alinhamento pélvico no plano frontal, estando o indivíduo na postura ereta), tais recursos não poderiam ser utilizados por não fornecerem dados suficientes para análise tridimensional da pelve. Além disso, esses exames são de custo elevado, muitas vezes, inacessíveis, principalmente na prática clínica, além de apresentarem um potencial risco pela radiação emitida 5,19,20,26. Portanto, métodos alternativos são desenvolvidos ${ }^{5,19}$ e utilizados com freqüência na prática diária dos profissionais e em estudos científicos da área ${ }^{22,26}$.

Tabela 1. Estatística descritiva (médias, desvios padrão e faixa de variação das medidas utilizadas para análise da correlação entre as variáveis investigadas $(\mathrm{n}=32)$. controle de determinadas características, como a experiência do examinador, a pressão aplicada sobre o tecido, a manutenção de um único examinador para realizar o procedimento e a quantidade de tecido adiposo sobre o local são essenciais para a acurácia desse procedimento ${ }^{26}$. Neste estudo, os examinadores praticaram exaustivamente a palpação das estruturas utilizadas como referências, procurando sempre utilizar a mesma técnica, e apenas um examinador ficou responsável por identificar determinada estrutura em todos os indivíduos avaliados. Além disso, as estruturas utilizadas são consideradas de fácil identificação pela palpação, principalmente em indivíduos não obesos, questão considerada como critério de inclusão $0^{8,20,22,26,29}$.

Considerando o que foi discutido, os testes e procedimentos utilizados são os meios existentes e preconizados pela literatura, até o presente momento, para se obterem as medidas

\begin{tabular}{lcc}
\hline \multicolumn{1}{c}{ Variável } & Média $( \pm$ DP) & Faixa de variação \\
\hline Altura da EIAS mais elevada $(\mathrm{cm})$ & $95,04 \pm 4,87$ & 85,9 a 105,2 \\
Altura da EIAS mais baixa $(\mathrm{cm})$ & $94,5 \pm 4,89$ & 85,4 a 104,8 \\
EIAS mais elevada menos EIAS mais baixa $(\mathrm{cm})^{*}$ & $0,54 \pm 0,44$ & 0 a 2,0 \\
CBITe & $22,98 \pm 5,51$ & 12 a 32,5 \\
CBITb & $22,09 \pm 4,62$ & 11 a 30 \\
CBITe menos CBITb * & $0,88 \pm 4,54$ & -8 a 11,75 \\
\hline
\end{tabular}

\footnotetext{
* Variáveis utilizadas para análise de correlação; EIAS: espinha ilíaca ântero-superior; EIPS: espinha ilíaca póstero-superior; CBITe: comprimento da banda iliotibial correspondente ao lado da espinha ilíaca ântero-superior mais elevada; CBITb: comprimento da banda iliotibial correspondente ao lado da espinha ilíaca ântero-superior mais baixa.
}

Sanders e Stavrakas ${ }^{19}$ propuseram um método indireto, utilizando cálculo trigonométrico para se obterem medidas de inclinação pélvica, o qual vem sendo utilizado e aprimorado por diversos estudos ${ }^{19,22,26}$. Em um deles, foi investigada a confiabilidade intra e interexaminador das medidas de inclinação pélvica fornecidas pelo cálculo trigonométrico, com a utilização de um instrumento (ISP) para auxiliar no posicionamento do indivíduo ${ }^{22}$, e valores aceitáveis de confiabilidade intra e interexaminador foram encontrados.

No presente estudo, utilizando os três recursos preconizados pela literatura, até o momento, para obtenção da inclinação pélvica (palpação, inclinômetro e ISP), o CCI intra-examinador obtido foi superior ao relatado nos estudos prévios $(\mathrm{CCI}=0,99)$. Justificamos esse resultado pelo elevado rigor metodológico adotado na realização dos testes e medidas. A palpação das estruturas, necessária durante grande parte da coleta dos dados, apesar de ser uma prática universalmente utilizada, tanto na prática clínica fisioterápica, quanto em pesquisas científicas ${ }^{8,20,22,26,29}$, é ainda bastante discutida. O necessárias para este estudo. Por serem de baixo custo e de fácil utilização, são comumente aplicados na avaliação clínica fisioterapêutica, porém sem o rigor metodológico e sem os equipamentos de auxílio (nível pélvico, inclinômetro, ISP) utilizados neste estudo, fatores que podem comprometer a acurácia das medidas obtidas no ambiente clínico. Portanto, apontamos a necessidade de se adotarem determinados controles e de se utilizarem equipamentos de auxílio durante a realização dos testes em ambientes clínicos para que as medidas sejam acuradas.

É importante ressaltar alguns procedimentos adotados que podem ter influenciado, direta ou indiretamente, as medidas obtidas. Um deles é o posicionamento da articulação subtalar em neutro, procedimento descrito por Gould III ${ }^{24}$, realizado na tentativa de evitar que alterações dos pés afetassem a altura das estruturas pélvicas. Entretanto, não foi encontrado, na literatura científica, estudo sobre a validade concorrente e confiabilidade desse método. Na realização do estudo piloto, as medidas pélvicas obtidas, utilizando esse 
procedimento, apresentaram uma alta confiabilidade e, por isso, foi decidido manter o mesmo protocolo durante a coleta dos dados, uma vez que alterações biomecânicas nas articulações subtalares podem gerar uma diferença funcional no comprimento dos membros, o que poderia interferir diretamente na medida da altura pélvica ${ }^{24}$, tornando-se, portanto, um fator de confusão. Entretanto, não se pode descartar, completamente, o fato de que o posicionamento da articulação subtalar em neutro, segundo descrições de Gould III $^{24}$, possa ter interferido indiretamente nas medidas obtidas na pelve.

Além dos testes, medidas e procedimentos utilizados neste estudo, outros fatores que podem ter interferido nos resultados encontrados devem ser apontados. Um deles é a composição da amostra utilizada. Como foram indivíduos sem nenhuma queixa de dor ou de qualquer outra alteração em tronco e membros inferiores, as medidas obtidas podem não ser representativas. A média dos valores para a altura da EIAS mais elevada foi de $95,04 \mathrm{~cm}( \pm 4,87)$ e para a mais baixa foi de $94,5 \mathrm{~cm}( \pm 4,89)$ resultando em uma pequena diferença entre uma EIAS e outra: $0,54 \mathrm{~cm}( \pm 0,44)$. O mesmo pôde ser observado nas variáveis relacionadas ao CBIT. Entretanto, a decisão de estudar uma amostra com essas características foi tomada com o objetivo de homogeneizála e de diminuir fatores que poderiam interferir, direta ou indiretamente, nos resultados obtidos, possibilitando, assim, a realização do estudo.

Outro fator a ser considerado é o posicionamento descrito na literatura para se obter o CBIT: decúbito lateral ${ }^{1,2,9,25}$. A forma classicamente descrita para se medir o CBIT pode não refletir o seu real comprimento ou a sua ação sobre as estruturas quando o indivíduo encontra-se na posição ortostática, uma vez que vários outros tecidos apresentam conexão com a BIT, como a fáscia dos músculos glúteo máximo e tensor da fáscia lata e a conexão fibrosa dos músculos bíceps femoral e vasto lateral pelo septo intermuscular lateral $^{3}$. Portanto, características dessas estruturas que não interferem diretamente sobre a BIT, estando o indivíduo em decúbito lateral, podem atuar quando o indivíduo se encontra em outra postura, como na posição ereta, ou quando realiza alguma atividade de forma dinâmica, como durante a marcha, gerando um comportamento diferenciado. Portanto, faz-se necessário encontrar uma forma de se avaliar o CBIT, considerando a sua relação com outros tecidos e os posicionamentos que são funcionais aos indivíduos. Além disso, deve-se ter cautela ao formular conclusões clínicas baseadas nessas medidas.

Por último, deve-se considerar e discutir o fato de que o CBIT não possua relação com o DP. É plausível considerar que apenas alteração no comprimento dessa estrutura não tenha influência sobre o posicionamento da pelve, uma vez que um grande número de músculos, ligamentos e algumas fáscias também se inserem ou se originam nessa região. Assim, a modificação de uma única característica, em determinada estrutura, pode não ser suficiente para provocar alterações significativas na pelve. Além disso, os músculos da articulação do quadril, dos quais grande parte se conecta na pelve, funcionam em uma cadeia cinética fechada e são peculiares devido às suas grandes áreas de fixação, seu comprimento e sua ampla secção transversa. Como a articulação do quadril permite uma grande amplitude de movimento, esses músculos têm a sua função modificada de acordo com a posição da articulação, podendo, inclusive, exercer funções antagônicas em diferentes posições articulares. Por isso, os resultados dos estudos variam segundo as condições de teste, devendo ser interpretados com cuidado: o melhor seria, na medida do possível, encontrar uma forma de examinar a ação desses músculos no contexto de suas funções específicas ${ }^{3}$. Sendo assim, o complexo da pelve deve ser considerado como um todo, e os fatores que apresentam potencial de modificar a sua estrutura devem ser avaliados conjuntamente, evitando-se, sempre que possível, justificar determinada alteração com um achado clínico que pode estar presente sem, no entanto, possuir relação direta com a alteração ou a disfunção presente.

Enfim, apesar de não ter sido encontrada relação entre o CBIT e o DP, esse resultado deve ser interpretado com cautela, pois diversos fatores podem tê-lo influenciado. Assim, faz-se necessária a realização de estudos com indivíduos que apresentem disfunções associadas ao desalinhamento pélvico para uma melhor definição e abrangência dos fatores que podem estar relacionados a essas desordens.

Agradecimentos: Aos professores Hans-Joachim Menzel e Mauro Heleno Chaves responsáveis pelo Laboratório de Biomecânica da Escola de Educação Física, Fisioterapia e Terapia Ocupacional da UFMG.

\section{REFERÊNCIAS BIBLIOGRÁFICAS}

1. Kendall FP, McCreary EK, Provance PG. Músculos provas e funções. $4^{\text {th }}$ ed. São Paulo: Manole; 1995.

2. Kisner C, Colby LA. Exercícios terapêuticos exercícios e técnicas. $3^{\text {th }}$ ed. São Paulo: Manole; 1998.

3. Norkin CC, Levangie PK. Articulações estruturas e função: uma abordagem prática e abrangente. $2^{\text {nd }}$ ed. Rio de Janeiro: Revinter; 2001.

4. Badii M, Shin S, Torreggiani WC, Jankovic B, Gustafson P, Munk PL, et al. Pelvic bone asymmetry in 323 study participants receiving abdominal CT scans. Spine. 2003; 28(12):13359.

5. Gurney B. Leg length discrepancy. Gait Posture. 2002; (15):195206.

6. Fann AV. The prevalence of postural asymmetry in people with and without chronic low back pain. Arch Phys Med Rehabil. 2002;(83):1736-8.

7. Sahrmann SA. Movement impairment syndromes of the hip. In: Sahrmann SA, editor. Diagnosis and treatment impairment syndromes. St. Louis Missouri: MOSBY; 2002. 
8. Hanada E, Kirby L, Mitchell M, Swuste JM. Measuring leglength discrepancy by the "iliac crest palpation and book correction” method: reliability and validity. Arch Phys Med Rehabil 2001; 82(7):938-42.

9. Ober FR. The role of the iliotibial band and fascia lata as a factor in the causation of low-back disabilities and sciatica. J Bone Joint Surg 1936; 18(1):105-10.

10. Reese NB, Bandy WD. Use of an inclinometer to measure flexibility of the iliotibial band using the Ober test and de modified Ober test: differences in magnitude and reliability of measurements. J Orthop Sports Phys Ther. 2003;33(6):326-230.

11. Orchard JW, Fricker PA, Abud AT, Mason BR. Biomechanics of iliotibial band friction syndrome in runners. Am J Sports Med. 1996; 24(3):375-9.

12. Gajdosik RL, Sandler MM, Marr HL. Influence of knee positions and gender on the Ober test for length of the iliotibial band. Clin Biomech. 2003;(18):77-9.

13. Abdulhadi HM, Kerrigan DC, La Raia PJ. Contralateral shoe lift: effect on oxygen cost of walking with immobilized knee. Arch Phys Med Rehabil. 1996; (77):670-2.

14. Kaufman KR, Miller LS, Sutherland DM. Gait assymetry in patients with limb-length inequality. J Ped Orthop. 1996; 16(2):144-50.

15. Knutson GA. Incidence of foot rotation, pelvic crest unleveling, and supine leg length alignment asymmetry and their relationship to self-reported back pain. J Man Phys Ther. 2002; 25(2):1-7.

16. Svensson HO, Vedin, A. A retrospective study of low back pain in 38 to 64 year-old women: frequency and occurrence and impact on medical services. Spine. 1988;13(5):548-552.

17. Waddell G, Somerville D, Henderson I, Newton, M. Objective clinical evaluation of physical impaiment in chronic low back pain. Spine. 1992; 17(6): 617-628.

18. Beattie P, Isaacson K, Riddle DL, Rothstein JM. Validity of derived measurements of leg-length differences obtained by use of a tape measure. Phys Ther. 1990;70(3):150-157.
19. Sanders G, Stavrakas P. A technique for measuring pelvic tilt. Phys Ther. 1981 Jan;61(1):49-50.

20. Alviso DJ, Dong GT, Lentell GL. Intertester reliability for measuring pelvic tilt in standing. Phys Ther. 1988;68(9): 1347-1351.

21. Levangie PK. The association between static pelvic asymmetry and low back pain. Spine. 1999 Jun 15;24(12):1234-42.

22. Raso P, Cruz W, Zarattini JA, Carneiro RL, Chagas MH. Confiabilidade intra e inter-examinador da mensuração da inclinação e amplitude de movimento da pelve. Anais do X Congresso Brasileiro de Biomecânica; 2003 3-6 junho: Ouro Preto, Brasil; 2003.

23. Gross MT, Burns CB, Chapman SW, Hudson JC, Curtis HS, Lehmann JRRJB. Reliability and validity of rigid lift and pelvic leveling device method in assessing functional leg length inequality. J Orthop Sports Phys Ther. 1998;27(4):285-94.

24. GouldIII J. Fisioterapia na ortopedia e na medicina do esporte. São Paulo: Manole; 1993.

25. Melchione WE, Sullivan MS. Reliability of measurements obtained by use of an instrument designed to indirectly measure iliotibial band length. J Orthop Sports Phys Ther. 1993 Sep;18(3):511-5.

26. Gajdosik R, Simpson R, Smith R, Fann AV. Pelvic tilt intratester reliability of measuring the standing position and range of motion. Phys Ther. 1985 Fev; 65(2):169-74.

27. Faria CDCM, Lima FFP, Teixeira-Salmela, LF. Testes de Ober e Ober modificado: um estudo comparativo e de confiabilidade. Fisiot Mov. 2005 Jul-Set; 18(3): 31-38.

28. Piva SR, Erhard RE, Childs JD, Hicks G, Al-Abdulmohsin H. Reliability of measuring iliac crest level in the standing and sitting position using a new measurement device. J Manip Physiol Ther. 2003 Set;26(7):437-41.

29. Hoyle DA, Latour M, Bohannon RW. Intraexaminer, interexaminer, and interdevice comparability of leg length measurements obtained with measurings tape and metrecom. J Orthop Sports Phys Ther. 1991 Dez ;14(6):263-8. 